PROCEEDINGS OF THE

AMERICAN MATHEMATICAL SOCIETY

Volume 125, Number 11, November 1997, Pages 3169-3176

S 0002-9939(97)03928-2

\title{
ETA-QUOTIENTS AND ELLIPTIC CURVES
}

\author{
YVES MARTIN AND KEN ONO
}

(Communicated by William W. Adams)

\begin{abstract}
In this paper we list all the weight 2 newforms $f(\tau)$ that are products and quotients of the Dedekind eta-function

$$
\eta(\tau):=q^{1 / 24} \prod_{n=1}^{\infty}\left(1-q^{n}\right),
$$

where $q:=e^{2 \pi i \tau}$. There are twelve such $f(\tau)$, and we give a model for the strong Weil curve $E$ whose Hasse-Weil $L$-function is the Mellin transform for each of them. Five of the $f(\tau)$ have complex multiplication, and we give elementary formulae for their Fourier coefficients which are sums of Hecke Grössencharacter values. These formulae follow easily from well known $q-$ series infinite product identities.
\end{abstract}

In light of the proof of Fermat's Last Theorem by A. Wiles and R. Taylor, there have been many expository articles describing the nature of the ShimuraTaniyama conjecture, the conjecture which asserts that every elliptic curve $E$ over $\mathbb{Q}$ is modular. This implies that the Hasse-Weil $L$-function of an elliptic curve $E$ with conductor $N$ over $\mathbb{Q}$,

$$
L(E, s)=\sum_{n=1}^{\infty} \frac{a(n)}{n^{s}}
$$

is the Mellin transform of a weight 2 newform $f(\tau) \in S_{2}(N)$ with Fourier expansion

$$
f(\tau)=\sum_{n=1}^{\infty} a(n) q^{n}
$$

where $q:=e^{2 \pi i \tau}$.

Many explicit examples of this correspondence have been given and it appears that the easiest examples to state correspond to those cases where the cusp form $f(\tau)$ is an eta-quotient. We have been encouraged by a number of people to write this short note where we once and for all compile the complete list of weight 2 newforms that are eta-quotients along with their strong Weil curves. In the next section we describe the method which produces this list of newforms, and we present the table of elliptic curves $E$ corresponding to these cusp forms. For the five curves with

Received by the editors March 2, 1996 and, in revised form, May 17, 1996.

1991 Mathematics Subject Classification. Primary 11F20, 11GXX.

Key words and phrases. Eta-quotient, elliptic curves.

The second author is supported by NSF grants DMS-9508976 and DMS-9304580.

(C)1997 American Mathematical Society 
complex multiplication, we show how the Grössencharacters are easily described by well known $q$-series infinite product identities.

We begin by exhibiting all weight 2 newforms with trivial Nebentypus character $f(\tau):=\prod \eta^{r_{i}}\left(t_{i} \tau\right)$, where $r_{i}$ are non-zero integers, and $t_{i}$ are positive integers. This is a special case of a more general result obtained in [10], where all such forms of arbitrary integral weight, level, and character that are Hecke eigenforms (plus a condition involving the Fricke involution) are classified.

Given positive integers $s, t_{1}, t_{2}, \ldots, t_{s}$, and integers $r_{1}, r_{2}, \ldots, r_{s}$, one considers the formal product $g=t_{1}^{r_{1}} t_{2}^{r_{2}} \ldots t_{s}^{r_{s}}$. Then $\eta_{g}(\tau)$ denotes the function on the upper half of the complex plane $\eta\left(t_{1} \tau\right)^{r_{1}} \eta\left(t_{2} \tau\right)^{r_{2}} \ldots \eta\left(t_{s} \tau\right)^{r_{s}}$. If this function is a holomorphic modular form we say that $\eta_{g}(\tau)$ is an $\eta$-quotient, and in those cases where all integers $r_{1}, \ldots, r_{s}$ are non-negative we call it an $\eta$-product.

Dummit, Kisilevsky and McKay [2] found all $\eta$-products which are newforms. In [10] this result was generalized, and it is determined that there are $74 \eta$-quotients $\eta_{g}(\tau)$ of integral weight such that both $\eta_{g}(\tau)$ and $\tilde{\eta}_{g}(\tau)$ (the image of $\eta_{g}(\tau)$ under the corresponding Fricke involution) are Hecke eigenforms. In this list all cusps forms turn out to be newforms.

These forms are interesting, among other things, because of their relations with representations of some sporadic simple groups. In [12] G. Mason related most of the $\eta$-products in [2] to the Mathieu group $M_{24}$ via some McKay-Thompson series. This relationship was extended in [13] using a generalized McKay-Thompson series. As for $\eta$-quotients, it is possible to show that at least 72 of the $74 \eta$-quotients classified in [11] can be associated to the Conway group $2 \mathrm{Co}_{1}$ (the automorphism group of the Leech lattice) via a particular generalized McKay-Thompson series (see [10], [11]).

Theorem 1. The following list contains all eta-quotients that are weight 2 newforms.

$\begin{array}{cc}\frac{\text { Conductor }}{11} & \frac{\text { Eta-quotient }}{\eta^{2}(\tau) \eta^{2}(11 \tau)} \\ 14 & \eta(\tau) \eta(2 \tau) \eta(7 \tau) \eta(14 \tau) \\ 15 & \eta(\tau) \eta(3 \tau) \eta(5 \tau) \eta(15 \tau) \\ 20 & \eta^{2}(2 \tau) \eta^{2}(10 \tau) \\ 24 & \eta(2 \tau) \eta(4 \tau) \eta(6 \tau) \eta(12 \tau) \\ 27 & \eta^{2}(3 \tau) \eta^{2}(9 \tau) \\ 32 & \eta^{2}(4 \tau) \eta^{2}(8 \tau) \\ 36 & \eta^{4}(6 \tau) \\ 48 & \frac{\eta^{4}(4 \tau) \eta^{4}(12 \tau)}{\eta(2 \tau) \eta(6 \tau) \eta(8 \tau) \eta(24 \tau)}\end{array}$




$$
\begin{array}{cc}
64 & \frac{\eta^{8}(8 \tau)}{\eta^{2}(4 \tau) \eta^{2}(16 \tau)} \\
80 & \frac{\eta^{6}(4 \tau) \eta^{6}(20 \tau)}{\eta^{2}(2 \tau) \eta^{2}(8 \tau) \eta^{2}(10 \tau) \eta^{2}(40 \tau)} \\
144 & \frac{\eta^{12}(12 \tau)}{\eta^{4}(6 \tau) \eta^{4}(24 \tau)}
\end{array}
$$

Proof (Sketch). First we observe that an $\eta$-quotient $\eta_{g}(\tau)$ of level $N_{g}$ and character $\chi_{g}$ has all its zeros at the cusps of $\Gamma_{0}\left(N_{g}\right)$. Their orders are given in terms of the parameters of $g=t_{1}^{r_{1}} t_{2}^{r_{2}} \ldots t_{s}^{r_{s}}$ by

$$
\frac{1}{24} \sum_{j=1}^{s} \frac{\operatorname{gcd}\left(t_{j}, c\right)^{2}}{t_{j}} r_{j}=\nu_{c} / h
$$

Here $\nu_{c}$ is the order of zero of $\eta_{g}(\tau)$ at the cusp $1 / c, h=h_{c}$ is the width of $1 / c$, and the character $\chi_{g}$ is assumed to be trivial.

The basic idea for the proof of the theorem is to show that the multiplicity of any zero (with respect to the local variable $q^{1 / h}$ ) of $\eta_{g}(\tau)$ is at most $2^{4}$, provided that the cusp form $\eta_{g}(\tau)$ is a newform.

This upper bound implies that only finitely many levels and weights are possible for such forms. By fixing the level $N_{g}$ we fix all parameters $t_{1}, t_{2}, \ldots, t_{s}$ of $g$, as the latter are divisors of the former. Next we observe that any set of possible multiplicities for the zeros of $\eta_{g}(\tau)$ (which are non-negative integers bounded by $2^{4}$ ), together with (1), defines a system of linear equations in the variables $r_{1}, r_{2}, \ldots, r_{s}$. An integral solution of this system (if any) determines a formal product $g$, and therefore an $\eta$-quotient $\eta_{g}(\tau)$. Clearly this process produces a finite list of modular forms containing all of those classified by the theorem, and now we just have to check which of these functions are indeed Hecke eigenforms.

Therefore the crux of the argument boils down to the computation of the upper bound described above. We now address this issue.

Let $f(\tau)$ be a modular form of level $N$, weight $k$ and Dirichlet character $\chi$ (for simplicity take $\chi$ to be trivial). Let $c$ be any factor of $N$. The Fourier series expansion of $f(\tau)$ at the cusp $1 / c$ is a power series of the form

$$
\left.f\right|_{k}\left(\begin{array}{ll}
1 & 0 \\
c & 1
\end{array}\right)=\sum_{n=\nu_{c}}^{\infty} a_{n} q_{h}^{n}, \quad \quad q_{h}=\exp (2 \pi i \tau / h)
$$

where $\nu_{c}$ is some non-negative integer with $a_{\nu_{c}} \neq 0$, and $h=h_{c}=N / \operatorname{gcd}\left(c^{2}, N\right)$ is the width of the cusp $1 / c$. The order or multiplicity of zero of $f(\tau)$ at $1 / c$ is the integer $\nu_{c}$.

Since every $\eta$-quotient has the property that the orders of vanishing at the cusps $1 / c$ and $a / c$ are the same if $\operatorname{gcd}(a, c)=1$, we may assume that $f(\tau)$ has this property.

If $p$ is any rational prime and $T_{p} f=\lambda_{p} f$ for some $\lambda_{p}$ in $\mathbb{C}$ one can relate the Fourier series of $f(\tau)$ at the cusps $1 / c$ and $1 / p c$. For example, if $p \mid N, \operatorname{gcd}\left(p, \frac{N}{p}\right)=1$ 
and $\operatorname{gcd}(p, c)=1$, there are integers $x^{\prime}, l^{\prime}$ and $n_{l}$ such that

$$
\left.p^{1-k / 2} \lambda_{p} f\right|_{k}\left(\begin{array}{ll}
1 & 0 \\
c & 1
\end{array}\right)=\left.\sum_{\substack{l=0 \\
l c \neq \equiv(p)}}^{p-1} f\right|_{k}\left(\begin{array}{cc}
1 & 0 \\
p c & 1
\end{array}\right)\left(\begin{array}{cc}
1 & 1-p n_{l} \\
0 & p
\end{array}\right)+\left.f\right|_{k}\left(\begin{array}{cc}
x^{\prime} & l^{\prime} \\
c & p
\end{array}\right)\left(\begin{array}{ll}
p & 0 \\
0 & 1
\end{array}\right)
$$

where $l^{\prime} c+1=x^{\prime} p$ and $l \equiv p n_{l}\left(\bmod \frac{N}{p c}\right)$. Similar relations exist in those cases where $\operatorname{gcd}(p, N)=1$ or $p^{M} \mid N$ with $\operatorname{gcd}\left(p, N / p^{M}\right)=1, M \geq 2$. More precisely, for the latter there is an equation relating

$$
\left.f\right|_{k}\left(\begin{array}{cc}
1 & 0 \\
p^{\alpha-1} c & 1
\end{array}\right) \quad \text { and }\left.\quad f\right|_{k}\left(\begin{array}{cc}
1 & 0 \\
p^{\alpha} c & 1
\end{array}\right)
$$

if $\frac{M+1}{2} \leq \alpha \leq M$

These identities are proved in the same way. This argument, however, does not work for $\alpha<\frac{M+1}{2}$. In order to get the same relations for these values of $\alpha$, we need to assume that $\tilde{f}(\tau)$, the image of $f(\tau)$ under the Fricke involution, is also an eigenform of $T_{p}$. This is the case if $f(\tau)$ is a newform and its Fourier coefficients at infinity are real numbers.

These equations yield some properties of the order of zero $\nu_{c}$. For example:

(i) $\nu_{c}=0$ or any prime divisor of $\nu_{c}$ is a factor of $\operatorname{gcd}(c, N / c)$.

(ii) If $p^{M} \mid N$ with $\operatorname{gcd}\left(p, \frac{N}{p^{M}}\right)=1, M \geq 3$, and $\operatorname{gcd}(p, c)=1$ then $\operatorname{gcd}\left(p, \nu_{p^{\alpha} c}\right)=1$ for every $0 \leq \alpha \leq M, \alpha \neq M / 2$.

(iii) Let $\eta(\tau)$ be a newform of level $N_{g}$ and trivial character $\chi_{g}$. Let $p^{M}$ be the p-part of $N_{g}$.

Then $M \geq 2$ implies $p=2$ or 3 . Furthermore $p=2$ implies $M \leq 8$, and $p=3$ implies $M \leq 3$.

(iv) Let $\eta_{g}(\tau)$ be as in (iii), and $2^{M}$ the 2-part of $N_{g}$.

Then $1 \leq \nu_{c} \leq 2^{M / 2}$ for every factor $c$ of $N_{g}$. Furthermore $\nu_{c}=1$ or $M$ is an even positive integer and $2^{M / 2}$ is the 2-part of $c$.

It should be noted that remarks (i), (ii) and (1) imply (iii). Although (1) only gives the smallest non-zero exponent of the variable $q$ in the Fourier series of $\eta_{g}(\tau)$ at $1 / c$, a careful examination of this power series (given also in terms of the parameters of $g$ ) yields (iv). By (iii) and (iv), $2^{4}$ is an upper bound for the orders of zero of such an $\eta$-quotient.

Now we give a corresponding modular elliptic curve $E$ over $\mathbb{Q}$, from the isogeny class of curves whose Hasse-Weil $L$-function agrees with the Mellin transform for each of the twelve weight 2 eta-quotient newforms. Since all these curves are known to be modular, the following theorem is easily verified by checking well known tables (see $[1])$.

If $E$ is an elliptic curve, then we shall label its coefficients $a_{i}$ as usual; namely they belong to the Weierstrass model

$$
E: y^{2}+a_{1} x y+a_{3} y=x^{3}+a_{2} x^{2}+a_{4} x+a_{6} .
$$

For more on the theory of elliptic curves see [6], [14], [15]. 
Theorem 2. The following table contains a strong Weil curve for each of the weight 2 newforms that are eta-quotients.

\begin{tabular}{|c|c|c|c|c|c|c|}
\hline Conductor & Eta-quotient & $\underline{a_{1}}$ & $\underline{a_{2}}$ & $\underline{a_{3}}$ & $\underline{a_{4}}$ & $\underline{a_{6}}$ \\
\hline 11 & $\eta^{2}(\tau) \eta^{2}(11 \tau)$ & 0 & -1 & 1 & -10 & -20 \\
\hline 14 & $\eta(\tau) \eta(2 \tau) \eta(7 \tau) \eta(14 \tau)$ & 1 & 0 & 1 & 4 & -6 \\
\hline 15 & $\eta(\tau) \eta(3 \tau) \eta(5 \tau) \eta(15 \tau)$ & 1 & 1 & 1 & -10 & -10 \\
\hline 20 & $\eta^{2}(2 \tau) \eta^{2}(10 \tau)$ & 0 & 1 & 0 & 4 & 4 \\
\hline 24 & $\eta(2 \tau) \eta(4 \tau) \eta(6 \tau) \eta(12 \tau)$ & 0 & -1 & 0 & -4 & 4 \\
\hline 27 & $\eta^{2}(3 \tau) \eta^{2}(9 \tau)$ & 0 & 0 & 1 & 0 & -7 \\
\hline 32 & $\eta^{2}(4 \tau) \eta^{2}(8 \tau)$ & 0 & 0 & 0 & 4 & 0 \\
\hline 36 & $\eta^{4}(6 \tau)$ & 0 & 0 & 0 & 0 & 1 \\
\hline 48 & $\frac{\eta^{4}(4 \tau) \eta^{4}(12 \tau)}{\eta(2 \tau) \eta(6 \tau) \eta(8 \tau) \eta(24 \tau)}$ & 0 & 1 & 0 & -4 & -4 \\
\hline 64 & $\frac{\eta^{8}(8 \tau)}{\eta^{2}(4 \tau) \eta^{2}(16 \tau)}$ & 0 & 0 & 0 & -4 & 0 \\
\hline 80 & $\frac{\eta^{6}(4 \tau) \eta^{6}(20 \tau)}{\eta^{2}(2 \tau) \eta^{2}(8 \tau) \eta^{2}(10 \tau) \eta^{2}(40 \tau)}$ & 0 & -1 & 0 & 4 & -4 \\
\hline 144 & $\frac{\eta^{12}(12 \tau)}{\eta^{4}(6 \tau) \eta^{4}(24 \tau)}$ & 0 & 0 & 0 & 0 & \\
\hline
\end{tabular}

Proof. This list is complete since all eigenforms that are weight 2 eta-quotients are known and are given in Theorem 1. These curves are all well known to be modular and they may be found in various tables (see [1]).

The curves with conductors $27,32,36,64$, and 144 have complex multiplication. Specifically the curves with conductors 27,36 , and 144 have complex multiplication by $\mathbb{Q}(\sqrt{-3})$ while the curves with conductors 32 and 64 have complex multiplication by $\mathbb{Q}(i)$. Moreover the curves with $N=36$ and 144 given in Theorem 2 are quadratic twists of each other by -1 .

In particular this implies that $L(E, s)$ is a Hecke $L$-function, a function corresponding to a Grössencharacter. First we recall essential preliminaries and definitions. Let $K=\mathbb{Q}(\sqrt{-d})$ be a quadratic imaginary field with integer ring $O_{K}$ with discriminant $-D$. A Hecke Grössencharacter $\phi$ of weight $k \geq 2$ with conductor $\Lambda$, an ideal in $O_{K}$, is defined in the following way. Let $I(\Lambda)$ denote the group of fractional ideals prime to $\Lambda$. We call a homomorphism $\phi: I(\Lambda) \rightarrow \mathbb{C}^{\times}$satisfying

$$
\phi\left(\alpha O_{K}\right)=\alpha^{k-1} \quad \text { when } \alpha \equiv 1 \quad \bmod \Lambda
$$


a Hecke Grössencharacter of weight $k$ and conductor $\Lambda$. The $L$-function $L(\phi, s)$ induced by a Hecke Grössencharacter is defined by

$$
L(\phi, s):=\sum_{\mathfrak{a}} \phi(\mathfrak{a}) N(\mathfrak{a})^{-s}=\sum_{n=1}^{\infty} \frac{a(n)}{n^{s}},
$$

where the sum is over ideals $\mathfrak{a} \subseteq O_{K}$ prime to $\Lambda$. Here $N(\mathfrak{a})$ is the ideal norm of $\mathfrak{a}$.

Deuring proved that if $E$ has complex multiplication, then $L(E, s)$ is a Hecke $L$-function $L(\phi, s)$ for suitable $\phi$. In all of the five cases where $E$ has complex multiplication we show how to find explicit formulae for the coefficients $L(\phi, s)$ using well known classical theta series due to Euler, Jacobi, Köhler, and Macdonald [7], [9].

The following $q$-series infinite product identities are well known:

(1. Euler)

(2. Jacobi)

(3. Jacobi)

$$
\begin{aligned}
\frac{\eta^{5}(2 \tau)}{\eta^{2}(\tau) \eta^{2}(4 \tau)} & =\prod_{n=1}^{\infty} \frac{\left(1-q^{2 n}\right)^{5}}{\left(1-q^{n}\right)^{2}\left(1-q^{4 n}\right)^{2}} \\
& =1+2 \sum_{n=1}^{\infty} q^{n^{2}}
\end{aligned}
$$

(4. Jacobi)

$$
\frac{\eta^{2}(\tau)}{\eta(2 \tau)}=\prod_{n=1}^{\infty} \frac{\left(1-q^{n}\right)^{2}}{\left(1-q^{2 n}\right)}=1+2 \sum_{n=1}^{\infty}(-1)^{n} q^{n^{2}}
$$

(5. Köhler-Macdonald)

$$
\frac{\eta^{5}(6 \tau)}{\eta^{2}(3 \tau)}=q \prod_{n=1}^{\infty} \frac{\left(1-q^{6 n}\right)^{5}}{\left(1-q^{3 n}\right)^{2}}=\sum_{n=1}^{\infty}(-1)^{n-1}\left(\frac{n}{3}\right) n q^{n^{2}}
$$

We now use these identities to compute the Fourier coefficients, which are sums of the relevant Hecke Grössencharacters, for those five forms with complex multiplication.

First we fix some notation. Let

$$
F_{N}(\tau)=\sum_{n=1}^{\infty} a_{N}(n) q^{n}
$$

be the weight 2 eta-quotient newform of level $N$. Since their coefficients are Hecke multiplicative, it suffices to give the formulae for $a_{N}(p)$ for $p$ prime; these are the traces of the Frobenius endomorphism. These formulae are the content of the following result. 
Theorem 3. In the notation above, we obtain the following formulae for primes $p \nmid N$ :

$$
\begin{aligned}
& a_{27}(p)=\left\{\begin{aligned}
0 & \text { if } p \equiv 2 \quad(\bmod 3), \\
2 m+n & \text { if } p \equiv 1 \quad(\bmod 3), p=m^{2}+m n+n^{2}, \\
& m \equiv 1 \quad(\bmod 3), n \equiv 0 \quad(\bmod 3),
\end{aligned}\right. \\
& a_{32}(p)= \begin{cases}0 & \text { if } p \equiv 3 \quad(\bmod 4), \\
(-1)^{n+m}(4 n+2) & \text { if } p \equiv 1 \quad(\bmod 4), \\
& p=(2 n+1)^{2}+4 m^{2}, n, m \geq 0\end{cases} \\
& a_{36}(p)= \begin{cases}0 & \text { if } p \equiv 2 \quad(\bmod 3), \\
2\left(\frac{n}{3}\right) n & \text { if } p \equiv 1 \quad(\bmod 3), p=n^{2}+3 m^{2},\end{cases} \\
& a_{64}(p)= \begin{cases}0 & \text { if } p \equiv 3 \quad(\bmod 4), \\
(-1)^{n}(4 n+2) & \text { if } p \equiv 1 \quad(\bmod 4), \\
& p=(2 n+1)^{2}+4 m^{2}, n, m \geq 0,\end{cases} \\
& a_{144}(p)=\left\{\begin{array}{lc}
0 & \text { if } p \equiv 2 \quad(\bmod 3), \\
2(-1)^{n-1}\left(\frac{n}{3}\right) n & \text { if } p \equiv 1 \quad(\bmod 3), p=n^{2}+3 m^{2} .
\end{array}\right.
\end{aligned}
$$

Proof. Except for $a_{144}(p)$, these results follow immediately from the $q$-series infinite product identities and Theorem 1 . The fact that the conductor 144 curve is a -1 quadratic twist of the conductor 36 curve immediately implies that for odd primes $p$

$$
a_{144}(p):=\left(\frac{-1}{p}\right) a_{36}(p)
$$

The result now follows from the formulae for $a_{36}(p)$.

The formula for $a_{27}(p)$ can be found in [8] and similar formulae for other multiplicative eta-products can be found in the works of Gordon, Hughes, Sinor, and Robins [3], [4], [5].

\section{ACKNOWLEDGEMENTS}

We thank the referee for making several suggestions which improved this note.

\section{REFERENCES}

1. J. E. Cremona, Algorithms for modular elliptic curves, Cambridge Univ. Press, Cambridge, 1992. MR 93m: 11053

2. D. Dummit, H. Kisilevsky, and J. McKay, Multiplicative properties of $\eta$-functions, Contemp. Math. 45, Amer. Math. Soc. (1985), 89-98.

3. B. Gordon and D. Sinor, Multiplicative properties of $\eta$-products, Springer Lect. Notes Math. 1395, Number Theory, Madras (1987), 173-200. MR 90k:11050

4. B. Gordon and S. Robins, Lacunarity of Dedekind $\eta$-products, Glasgow Math. J. (1995), 1-14. MR 96d:11044 
5. B. Gordon and K. Hughers, Multiplicative properties of $\eta$-products II, Contemp. Math. 143, Amer. Math. Soc. (1993), 415-430. MR 94a:11058

6. N. Koblitz, Introduction to elliptic curves and modular forms, Springer-Verlag, New York, 1984. MR 86c: 11040

7. G. Köhler, Theta series on the theta group, Abh. Math. Sem. Univ., Hamburg 58 (1988), 15-45. MR 90m: 11067

8. G. Köhler, Theta series on the Hecke groups $G(\sqrt{2})$ and $G(\sqrt{3})$, Math. Z. 197 (1988), 69-96. MR 89b: 11040

9. I. G. Macdonald, Affine root systems of Dedekind's $\eta$-function, Invent. Math. 15 (1972), 91-143. MR 50:9996

10. Y. Martin, Multiplicative eta-quotients, Trans. Amer. Math. Soc. 348 (1996), 4825-4856. MR 97d:11070

11. Y. Martin, On Hecke operators and products of the Dedekind $\eta$-function, C.R. Acad. Paris, 322 (1996), 307-312. MR 97a:11068

12. G. Mason, $M_{24}$ and certain automorphic forms, Contemp. Math. 45, Amer. Math. Soc. (1985), 223-244. MR 87c:11041

13. G. Mason, On a system of elliptic modular forms attached to the large Mathieu group, Nagoya Math. J. 118 (1990), 177-193. MR 91h:11038

14. J. Silverman, The arithmetic of elliptic curves, Springer-Verlag, New York, 1986. MR 87g:11070; MR 95m:11054

15. J. Silverman, Advanced topics in the arithemtic of elliptic curves, Springer-Verlag, New York, 1994. MR 96b:11074

Department of Mathematics, University of California, Berkeley, California 94720

E-mail address: ymartin@math.berkeley.edu

School of Mathematics, Institute for Advanced Study, Princeton, New Jersey 08540

E-mail address: ono@math.ias.edu

Department of Mathematics, The Pennsylvania State University, University Park, Pennsylvania 16802

E-mail address: ono@math.psu.edu 